\title{
Zur Polychaetenfauna des Eulitorals der Nordseeinsel Sylt
}

\author{
WILFRIED WEST'HEIDE \\ II. Zoologisches Institut und Museum der Universität, Göttingen ${ }^{1}$
}

\begin{abstract}
On the polychaete fauna of the eulitoral of the North Sea island Sylt. In the northern part of the island Sylt, 45 polychaete species, including archiannelids, were found between 1962 and 1965. These species live in 5 different habitats (biotopes): (1) lotic (exposed to intensive wave action), (2) semi-lotic and (3) lenitic (almost no wave action) sandy beaches, (4) sandy and (5) sandy-muddy mudflats. The relative abundance in these 5 habitats is indicated in Table 1.
\end{abstract}

\section{EINLEITUNG}

Bei Untersuchungen an psammobionten Vertretern der Gattungen Hesionides und Micropbthalmus (Westheide \& Ax 1965, Westheide 1966, Ax 1966) wurden in den Jahren 1962 bis 1965 zahlreiche Beobachtungen über die Polychaetenfauna im Eulitoral der Insel Sylt gemacht. Die faunistischen Befunde an dieser Tiergruppe sind hier mit dem Ziel zusammengestellt worden, für ein begrenztes Gebiet der Nordsee ein möglichst vollständiges Bild ihrer Verteilung zu geben. In der folgenden Übersicht werden deshalb neben den kleinen mesopsammalen Arten auch alle größeren Polychaeten angeführt. Letztere sind teilweise schon durch die Arbeit von WoHLENBERG (1937) für den Königshafen bekannt.

Die Durchführung der Arbeit erfolgte in der Litoralstation der Biologischen Anstalt Helgoland in List auf Sylt. Das Untersuchungsmaterial wurde aus folgenden Biotopen entnommen (Abb. 1):

(1) Breite lotischeS a nd strände: Weststrand (St. 1).

(2) Breite fast-1otische Sandstrände: Ellenbogen-Südstrand (St. 2); Litoralstation-Oststrand (St. 4).

(3) Schmale lenit ische Sand strände: Lister Haken (St. 3); Munkmarsch (St. 5); Rantum-Oststrand (St. 6).

(4) S a ndige Wattge biete mit relativ geringem Detritusgehalt: EllenbogenSüdstrand (St. 2); Litoralstation-Oststrand (St. 4).

(5) Breite sandig-sch lickige Wa t t f lä ch e n : Lister Haken - Königshafen (St. 3); Munkmarsch (St. 5).

1 Neue Anschrift: Biologische Anstalt Helgoland, Meeresstation, Helgoland. 
Von den Wattbiotopen ist nur ein ungefähr $10 \mathrm{~m}$ breiter, dem Sandstrand vorgelagerter Streifen eingehender bearbeitet worden. Alle übrigen Lebensräume habe ich zum Teil im Ablauf eines ganzen Jahres qualitativ und quantitativ in ihrer Zusammensetzung untersucht.

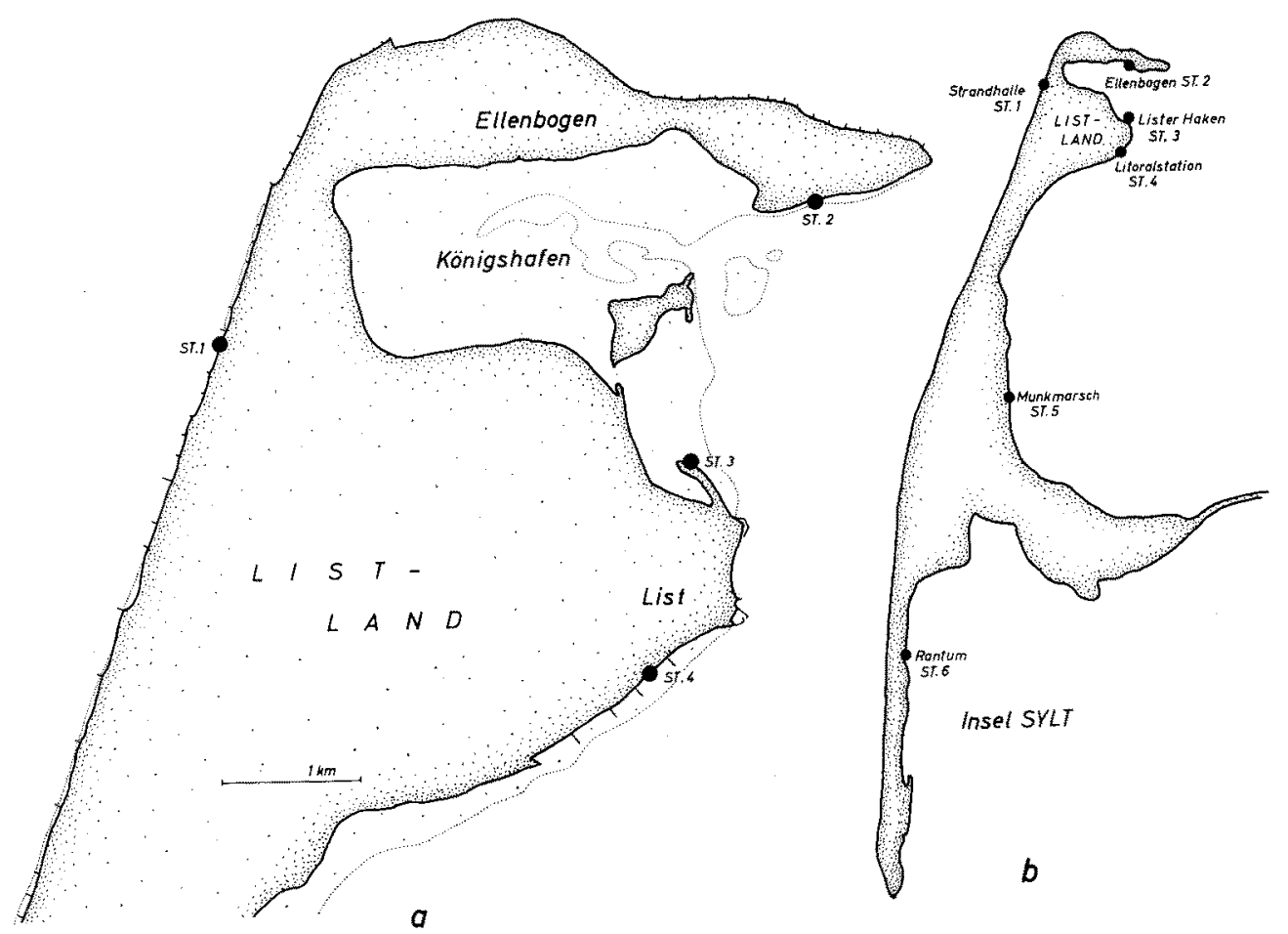

Abb. 1: Insel Sylt: Fundortsstationen. (a) Nordteil der Insel (Listland); (b) Gesamtumriß der Insel

\section{ERGEBNISSE}

45 Polychaetenarten wurden nachgewiesen; sie sind in Tabelle 1 nach ihrer Familienzugehörigkeit zusammengestellt worden. Bei Hesionides maxima, Microphthalmus listensis und Parapodrilus psammopbilus handelt es sich um für die Wissenschaft neue Arten, die aber bereits anderweitig beschrieben wurden (WestheIDE 1965, 1966). Micropbthalmus aberrans und Microphthalmus similis repräsentieren neue Elemente für die deutsche Fauna. Spbaerodoridium cf. balticum ist im deutschen Küstenbereich bislang nur aus der Ostsee bekannt. Stygocapitella subterranea, Streptosyllis bidentata und Aricidea suecica werden für die Nordsee von Remane (1955) erwähnt, erhalten jedoch hiermit erstmalig definierte Fundortsangaben.

Okologisch können wir Hesionides arenaria, Stygocapitella subterranea und Tri- 
lobodrilus ${ }^{2}$ als typische Bewohner des Prallhanges bezeichnen. Charakterarten des detritusarmen Sandwatts sind Micropbthalmus listensis, Psammodrilus balanoglossoides und Protodrilus symbioticus. Scolelepis squamata, Ophelia ratbkei und Protodrilus chaetifer treten besonders in der Übergangszone (Quellregion) zwischen beiden Lebensräumen auf. Mit zunehmendem Detritusgehalt und dem Übergang zu sandigschlickigen Biotopen wächst die Zahl der typischen Wattpolychaeten wie Gattyana cirrosa, Nephtys hombergi, Scoloplos armiger, Pygospio elegans, Heteromastus filiformis, Arenicola marina, Lanice conchilega.

Die Hesionidae stellen mit 6 Arten neben den Spionidae (4 Arten) die artenreichste Familie im Untersuchungsbereich. Quantitativ dominieren die Arten Trilobodrilus spec., Ophelia ratbkei und Hesionides arenaria mit Mengen von 3,4 Millionen, 1,8 Millionen bzw. 33000 Individuen auf $1 \mathrm{gm}$ einer $1 \mathrm{~cm}$ hohen Oberflächenschicht im unteren Prallhang. Derartige Mengen treten nur in den Sommermonaten auf. Bei Ophelia handelt es sich in dieser Zeit um Jungtiere, deren Zahl im Laufe des Winters stark reduziert wird. Trilobodrilus und Hesionides verlassen die oberflächlichen Schichten des Sandstrandes im Winter und sind dann nur in tieferen Bereichen in geringer Zahl zu finden (WestheIde 1966).

Die allgemeine Verbreitung der schon bekannten Arten ist besonders bei FRIEDrICH (1938) und REMANE (1932) zusammengestellt. Ich gehe deshalb nur auf die für unseren Faunenbereich neuen Elemente ein.

Streptosyllis bidentata, 1914 von der Irischen Küste beschrieben, ist im Sylter Eulitoral nicht selten, jedoch handelt es sich wahrscheinlich nicht um eine biotopeigene Art; der Hauptlebensraum dürfte in anderen, möglicherweise sublitoralen Bereichen zu suchen sein.

Sphaerodoridium of. balticum ist der einzige Vertreter der Sphaerodoridae. Habitus, Borsten und Zahl der Hautkapseln stimmen mit der aus der Ostsee beschriebenen Art überein; die Form der Parapodien weist dagegen gewisse Unterschiede auf. Da die Typen von Remers (1933) nicht mehr auffindbar sind, kann die Frage nach der Artidentität nur über neues Vergleichsmaterial aus der Ostsee entschieden werden.

Der Nachweis von Aricidea suecica und Stygocapitella subterranea auch für die Nordsee bestätigt die Ansicht von Friedrrch (1938), daß es sich bei diesen Formen nicht um endemische Arten handelt.

Stygocapitella betrachten wir darüber hinaus wie Hesionides arenaria als kosmopolitischen Sandlückenbewohner (Schwarzes Meer: Valkanov 1954; französische Atlantikküste: Renaud-Debrser 1963). Als weitere Fundorte gebe ich hier die französische Mittelmeerküste an (Etang de Salces, bei Le Barcarès); von der französischen Pazifikküste (San Juan Island, False Bay, Washington) liegt ein Exemplar vor, das auf Grund von Borstenbewaffnung und Segmentzahl ebenfalls zur Gattung Stygocapitella zu stellen ist. Dieses Material wurde mir von Herrn Prof. P. Ax überlassen, dem ich dafür besonders danke.

Microphthalmus aberans ist wahrscheinlich nur durch seine großen habituellen Übereinstimmungen mit $M$. sczelkowii bisher in der deutschen Fauna übersehen wor-

${ }^{2}$ Die im Sylter Eulitoral gefundene Trilobodrilus-Art ist nicht mit $T$, beideri ReMANL: aus dem Sublitoral von Helgoland identisch. Eine Bearbcitung der Gattung ist vorgesehen. 


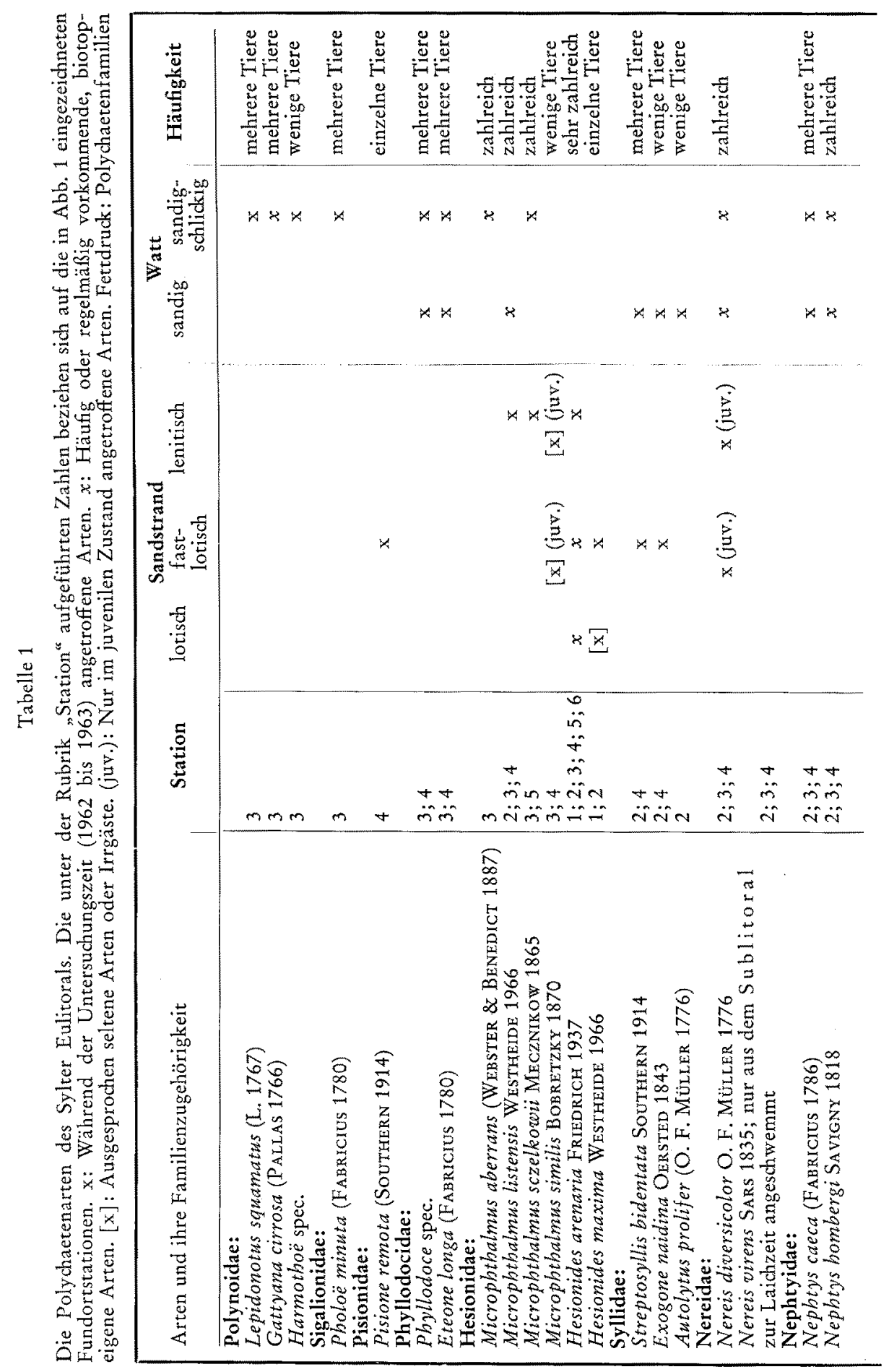




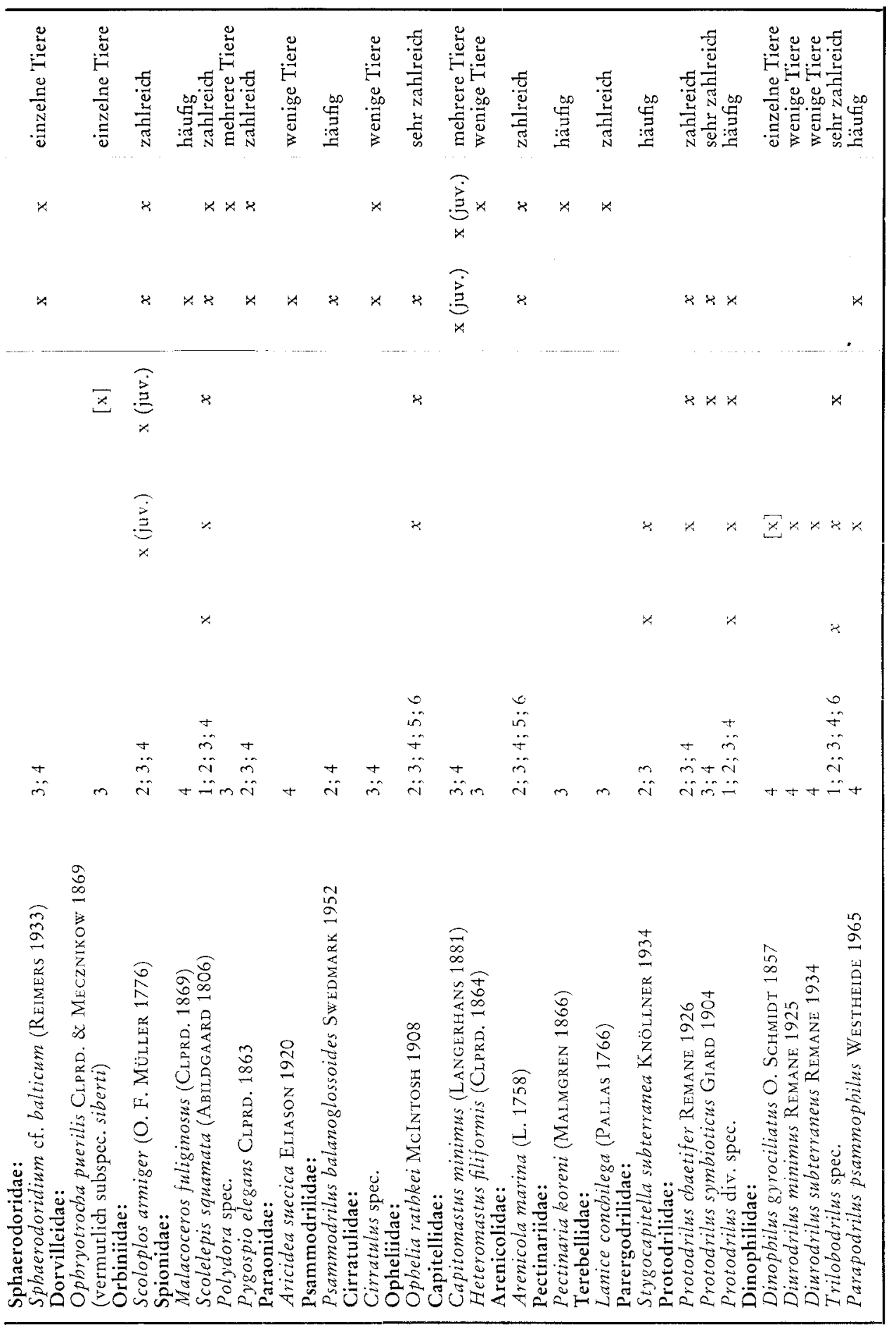


den (FrIEDRICH 1938, p. 101). Die wenigen juvenilen Exemplare von Microphthalmus similis halte ich für Irrgäste, die wohl als pelagische Larven an die deutsche Küste verschlagen worden sind.

\section{ZUSAMMENEASSUNG}

1. Die Polychaetenfauna der eulitoralen Prallhang- und Wattbiotope des Nordteils der Insel Sylt (Nordsee, Deutsche Bucht) umfaßt 45 Arten. Verbreitung und Häufigkeit in den einzelnen Lebensräumen des Untersuchungsgebietes wurden in einer Tabelle dargestellt.

2. Neue Arten sind Hesionides maxima WeSTHEIDE (1966), Microphthalmus listensis Westheide (1966) und Parapodrilus psammophilus Westheide (1965). Als neue Elemente für die deutsche Fauna wurden Microphthalmus aberrans (WEBsTER \& BeNEDICT 1887) und Microphthalmus similis BOBRETZKY (1870) festgestellt. Für den Bereich der Deutschen Bucht ist Sphaerodoridium cf. balticum (REImERs 1933) erstmalig beobachtet worden; für Streptosyllis bidentata Southern (1914), Aricidea suecica Eliason (1920) und Stygocapitella subterranea KNölLnER (1934) werden Fundorte in der Deutschen Bucht genannt.

Herrn Prof. Dr. O. Kinne, Direktor der Biologischen Anstalt Helgoland, und Herrn Dr. H. J. Aurich schulde ich Dank für die freundliche Aufnahme in der Litoralstation der Biologischen Anstalt Helgoland in List auf Sylt. Frau Dr. G. Hartmann-Schröder, Hamburg, und Herrn Dr. E. ZregelmeIER, List, danke ich für wichtige Hinweise, meinen Kommilitonen T. TEUchert, J. DörJes und P. SChmidt für die Uberlassung faunistischer und ökologischer Angaben.

\section{ZITIERTE LITERATUR}

Ax, P., 1966. Die Bedeutung der interstitiellen Sandfauna für allgemeine Probleme der Systematik, Okologie und Biologie. Veröff. Inst. Meeresforsch. Bremerh. (9) Sonderbd 2, 15-65.

Bobretzkx, N., 1870. On the fauna of the Black Sea [Russ.]. Zap. Kiev. Obshch. Estest. 1, $1-26$.

Eliason, A., 1920. Biologisch-faunistische Untersuchungen aus dem Oresund. 5. Polychaeten. Lunds Univ. Arsskr. N. F. (Avd. 2) 16, 1-103.

Friedrich, H., 1938. Polychaeta. In: Die Tierwelt der Nord- und Ostsee. Hrsg. von G. Grimpe \& A. E. Wagler. Akad. Verl. Ges., Leipzig, T. 6b, 1-201.

KNölliner, F., 1934. Die Tierwelt des Küstengrundwassers bei Schilksee (Kieler Bucht). 5. Stygocapitella subterranea n. gen. n. spec. Schr. Naturw. Ver. Scblesw.-Holst. 20, $468-472$.

Rermers, H., 1933. Morphologie der Polychaetengattung Sphaerodorum. Monographie. Zool. Jb. (Syst. Ökol. Geogr. Tiere) 64, 41-110.

Remane, A., 1932. Archiannelida. In: Die Tierwelt der Nord- und Ostsee. Hrsg. von G. Grimpe \& A. E. Wagler. Akad. Verl. Ges., Leipzig, T. 6a, 1-36.

- 1955. Die Brackwasser-Submergenz und die Umkomposition der Coenosen in Belt- und Ostsee. Kieler Meeresforsch. 11, 59-73.

Renaud-Debyser, J., 1963. Recherches écologiques sur la faune interstitielle des sables (Bassin d'Arcachon, ̂̂le de Bimini, Bah.). Vie Milien (Suppl.) 15, 1-157. 
Southern, R., 1914. Archiannelida and Polychaeta. (Clare Island survey. Pt 47.) Proc. R. Ir. Acad. 31, 1-160.

Valkanov, A., 1954. Zwei neue Polychaeten für das Schwarze Meer [Bulgar.]. Trud. chernomorsk. biol. Sta. Varna 18, 55-58.

Webster, H. E. \& Benedict, J. E., 1887. The Annelida Chaetopoda from Eastport, Maine. Rep. U.S. Commnr Fish. 13 (1885), 707-755.

WestheIDE, W., 1965. Parapodrilus psammophilus nov. gen. nov. spec., eine neue PolychaetenGattung aus dem Mesopsammal der Nordsee. Helgoländer wiss. Meeresunters. 12, 207-213.

- 1966. Monographie der Gattungen Hesionides FrIedrich und Micropbthalmus MeczniKow (Polychaeta, Hesionidae). Ein Beitrag zur Organisation und Biologie psammobionter Polychaeten (Math.-nat. Diss., Göttingen).Z. Morph. Okol. Tiere 57 (im Druck).

- \& Ax, P., 1965. Bildung und Obertragung von Spermatophoren bei Polychaeten (Untersuchungen an Hesionides arenarius FrIEDrICH). Zool. Anz. (Suppl. Bd) 28, 196-203.

Wohlenberg, E., 1937. Die Wattenmeer-Lebensgemeinschaften im Königshafen von Sylt. Helgoländer wiss. Meeresunters. 1, 1-92. 